\title{
The Research of NC Programming Method Based on DXF File
}

\author{
Qisheng Liu ${ }^{1,}$, Dechen Huang ${ }^{2, \mathrm{~b}}$, Xueli Tai ${ }^{3, \mathrm{c}}$, Tao Han ${ }^{4, \mathrm{~d}}$ and Bingbing Yan ${ }^{*, \mathrm{e}}$ \\ Jiamusi University, Jiamusi, Heilongjiang, 154007, China \\ aqsh815@sohu.com, b2292443911@qq.com, 'lucktxl@126.com, dhantao1969@163.com \\ eyanbingbing@126.com
}

*corresponding author: yanbingbing@126.com

\section{Keywords: Drawing Exchange File, Numerical Control Programming, CAD/CAM.}

\begin{abstract}
DXF format file is a standard format of the ASCII text file, the CAD graphic data is saved accurately. A method of NC program is proposed based on DXF file in this paper, the valid data is extracted from AutoCAD DXF document in the graphics file (including straight line, circle, circular, elliptic arc, and other information), then the design method of object-oriented program is used, thus the $\mathrm{NC}$ code can be identified by $\mathrm{NC}$ machine tools. A feasible way is provided to achieve the integration of CAD/CAPP/CAM system.
\end{abstract}

\section{Introduction}

With the rapid development of modern economy, integration technology of CAD/CAM has become more and more important in enterprise. One of the most important links in this technology is how to extract and convert the drawing information from CAD to CAM information. As a complete graphics editing system, AutoCAD has been a leading role in CAD field, but DWG file has a compact binary system which makes user hard to utilize the data information in the drawing directly. Therefore, a universal ASCII file is provided which equals to DWG file by Autodesk Company, it's called Drawing Exchange File (DXF). Nowadays, DXF has become one of standard data exchanging files in CAD field. Mainstream CAD software is compatible with DXF. The workload of converting CAD to CAM can be reduced by making use of parts drawing data in DXF to generate $\mathrm{CNC}$ machining program. Thus, the efficiency of design and production can be improved a lot. Recently, computer technology is applied widely in engineering field which leads to the secondary development with $\mathrm{VC}++$ etc. Object-oriented language becomes one of the hot issues. DXF file format is introduced by Chen $1 \mathrm{i}^{[1]}$, and thus AutoCAD external data framework is deduced, the interface of DXF data file is researched by Ziru Wang ${ }^{[2]}$ based on $\mathrm{VC}++$. On the basis of analyzing DXF, Naiwang MU ${ }^{[3]}$ realizes automatic programming of CNC EDM wire-cutting. However, tracks of wire-cutting machines usually are involved with two kinds of metafile of line and arc. And extracting and converting of other metafiles $\mathrm{CNC}$ code not be mentioned. On the basis of analyzing DXF format, the extracting and converting method of foregoing metafiles is provided in this paper. A NC-Presses is taken for an example to provide development processes and results of $\mathrm{VC}++$ applications.

\section{DXF File Format}

DXF format is a kind of representation method which contains marking data of all the information in AutoCAD drawing file. Marking data means that there is an integral number called group code before each data element ${ }^{[4]}$. Group code and its subsequent value always come in pairs, and group code and its value all take one line respectively. DXF file consists of 6 sections, they are header section, classes section, tables section, blocks section, entitles section and objects section. Each section begins with a "section" and a following " 0 ", ends with a "ENDSEC" and a following " 0 ". The content between them consists of defined metafile/object group code and value. The main information in each section refers to document ${ }^{[2]}$. In DXF, metafile (such as point, line, circle, arc, elliptic arc, polyline etc.) can be found in BLOCKS and ENTITLES section. Following will be 
given the meaning of each group-code in two-dimensional coordinate, and provide technical support for the converting of NC programs.

\section{Metafile Information Processing}

\section{DXF File and Interface of Higher-Level Language.}

CAD has some advantages on results display, editing and output. High-Level Language has some advantages on numerical calculation, numerical analysis. When needed to have a further processing for the generated drawing files, drawing system and High-Level Language Interface is the key to solve problems. The interface program of this DXF file is mainly developed with VC++ visualization program. With the application of interface program can get the metafile information of drawing in DXF file quickly. Mapping the drawing or outputting metafile information according to the loaded metafile data in order to demand designer.

\section{Loading metafile data.}

Because the key of this system is to load the geometry information and data of metafile in the drawing file. In each section of DXF drawing file, because entity section contains all of pixel information such as point coordinate, line coordinates, centre of arc, radius and flare angle etc. So, system can ignore the information be saved in other sections, only load entity section and End Of File. One entity section corresponds to one type of metafile as shown in Table 1. For getting metafile information, Higher-Level Language is used basically to a known ASCII file for reading and writing operation. By calculating the related data and get the final result to realize drawing processing. Arc-processing is taken for an example to demonstrate how to load metafile information as shown in Fig.1.

Table 1 Figure meaning tuple code

\begin{tabular}{ccll}
\hline Primitives Name & Variable Name & Tuple Code & \multicolumn{1}{c}{ Meaning } \\
\hline Point & AcDbPoint & $(1020)$ & $\mathrm{x}$ and y coordinates \\
\hline \multirow{2}{*}{ Line } & AcDbLine & $(1020)$ & $\begin{array}{l}\text { origin } \mathrm{x} \text { and } \mathrm{y} \text { coordinates } \\
\text { terminal } \mathrm{x} \text { and y coordinates }\end{array}$ \\
\hline \multirow{2}{*}{ Circle } & AcDbCircle & $(1121)$ & centre of a circle coordinates \\
& & $(40)$ & radius \\
\hline \multirow{2}{*}{ Arc } & & $(1020)$ & centre of a circle coordinates \\
& AcDbArc & $(40)$ & radius \\
& & $(50)$ & origin angle \\
& & $(51)$ & terminal angle \\
\hline \multirow{2}{*}{ Ellipse } & $(1020)$ & centre of a circle coordinates \\
& & $(1121)$ & long axis x and y coordinates \\
& AcDbEllipse & $(40)$ & ratio of long axis and short axis \\
& & $(41)$ & origin parameters \\
& & $(42)$ & terminal parameters \\
\hline
\end{tabular}




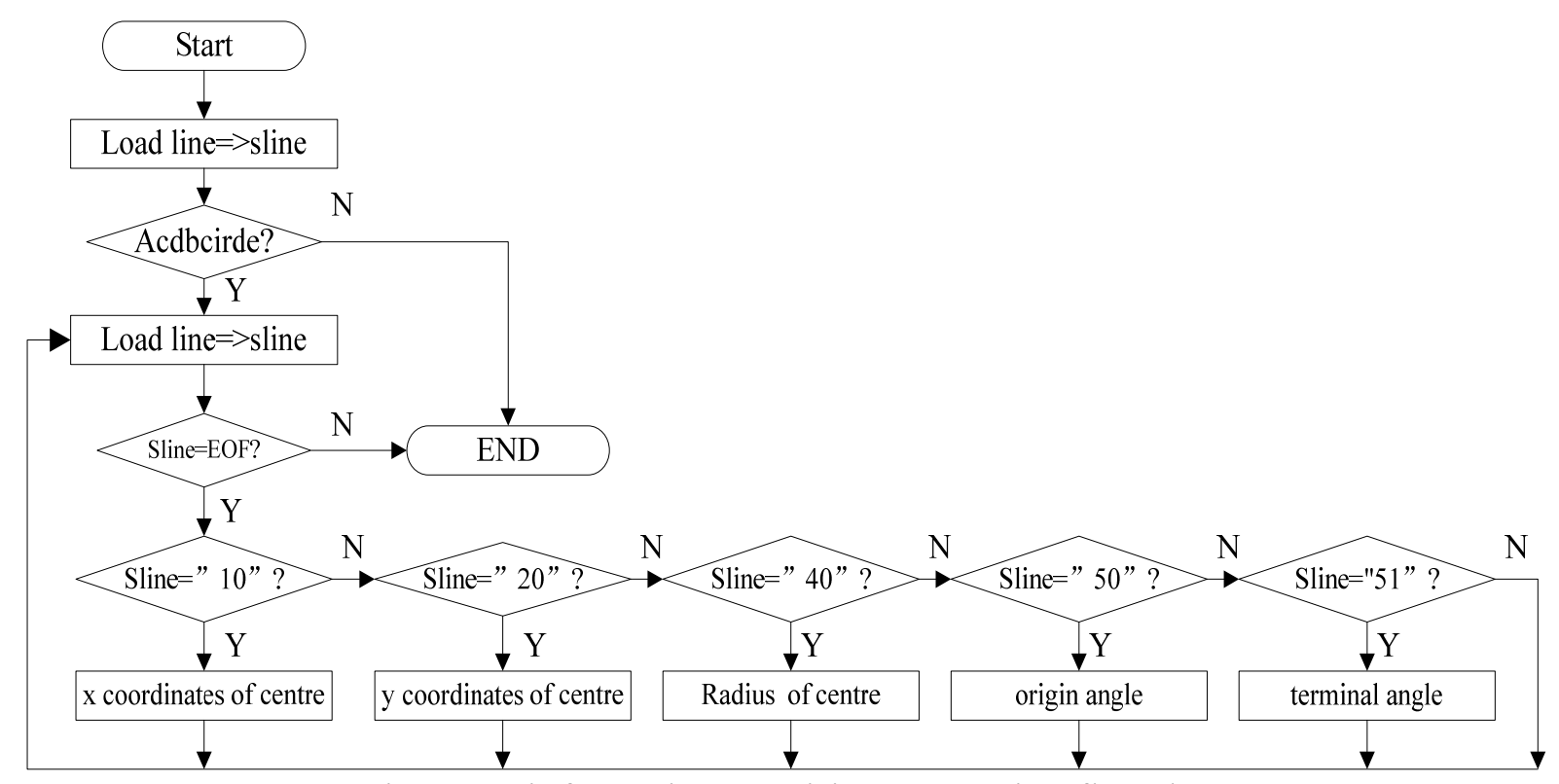

Fig.1 Arc information acquiring processing flowchart

\section{Realization Procedures}

Loading DXF parts drawing files to convert location coordinates data should take relevant process knowledge into consideration. To generate $\mathrm{NC}$ machining program, if need confirm some information such as cutter numbers, processing methods by operator, man-machine interaction can be adopted. In order to reduce the path length, a path optimization algorithm is proposed based on genetic algorithm by Yanqing Liang ${ }^{[5]}$. Post-processing is a text editor process, its function is loading the drawing information according to specified NC machine characteristics and specified format, then analyzing, judging and processing, converting into the machine-identification processing code and output.

Taking NC punch for an example to show the generation process of NC codes. Take $4 \mathrm{~mm} * 4 \mathrm{~mm}$ square cutter to punch $30 \mathrm{~mm} * 40 \mathrm{~mm}$ rectangle. How to choose rectangle processing methods is shown in Fig.2. The tree of rectangle generation is shown in Fig.3.

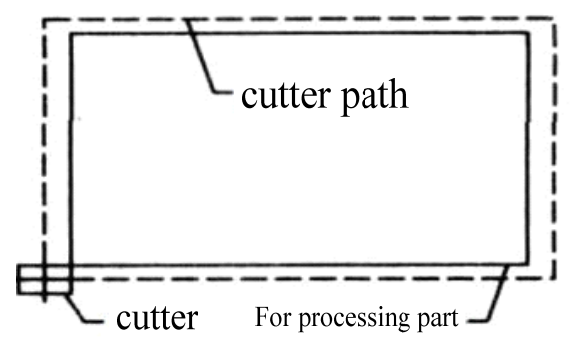

a) Lateral Processing

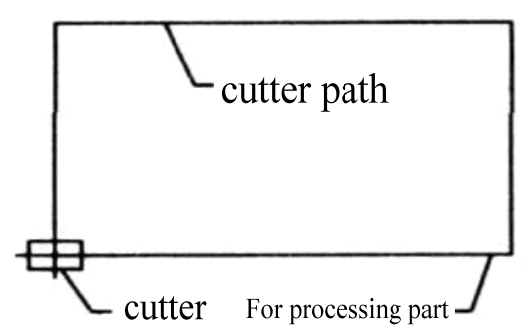

b) Standard Processing

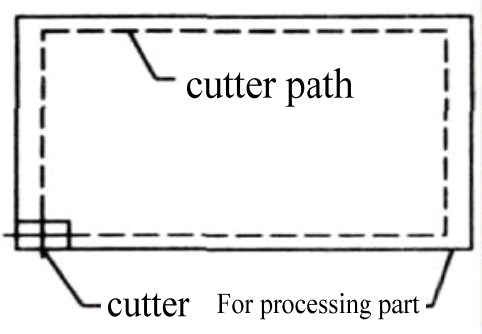

c) Medial Processing

Fig.2 Rectangle processing methods 


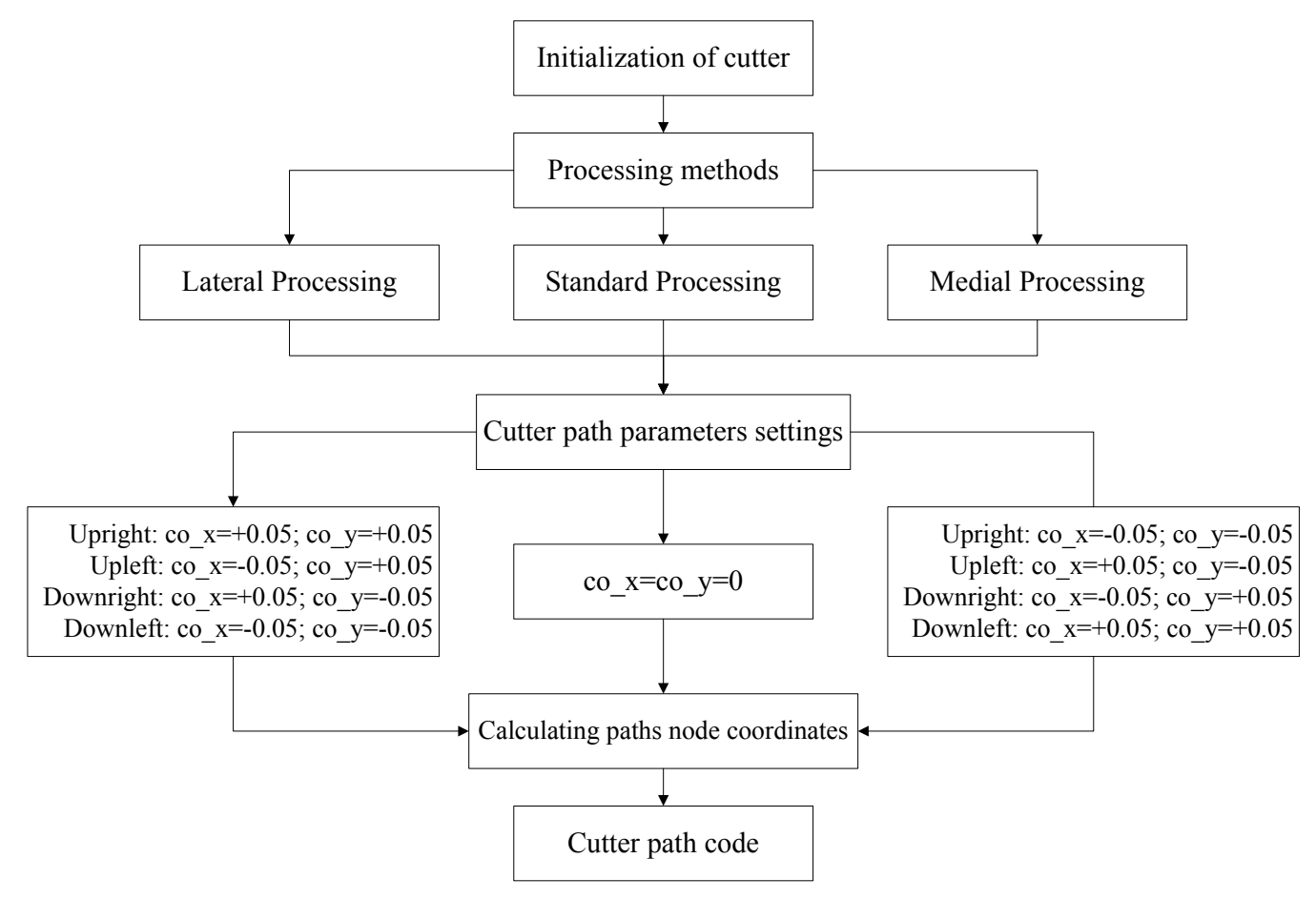

Fig.3 Rectangular machining program flowchart

\section{Summary}

Usually, designers draw on computers, the drawing data is read by technologists, the codes and process are written by NC machine. DXF is a standard format of the ASCII text files, and the CAD graphics Data is saved accurately. The graphical interface program can be extracted by a number of high-level languages to realize conversion between CAD graphics and CAM NC code, CAM processing procedures are generates by combing with numerical control technology knowledge. So the characteristics and advantages of DXF is the integration of CAD and CAM.

\section{Acknowledgments}

This work has been supported by the Science and Technology Research Project of Jiamusi University (L2009-117).

\section{References}

[1]. Chen li. Implementation of Data Interface to DXF Format in AutoCAD Using Visual C++. Industrial Control Computer. Vol. 23(2010) No. 4, p. 76-77.

[2]. Ziru Wang, Qingbo Ren. Research on DXF Data File Interface Based On VC++ Programming. Journal of Xiamen University of Technology. Vol. 15 (2007) No. 1, p. 26-30.

[3]. Naiwang $\mathrm{Mu}$, Yongdong Zhao and Zhongchen Shan. Automatically Programming of CNC Wire -out EDM Machine Based on DXF Files. Applied Science and Technology. Vol. 28(2001) No. 4, p. 1-3.

[4]. Zhanlong Yu. The Data form Conversion Between Softwares Based on CAD. Journal of Jiamusi University (Natural Science Edition). Vol. 20(2002) No. 1, p. 99-101.

[5]. Yanqing Liang, Wenjiang Wu, Pin Wang. Path Optimization in CNC Automatic Programming Based on DXF Files. Computer Systems \& Applications. Vol. 26(2015) No. 6, p. 173-176. 